\title{
CENU treatment induced bystander effects which are effective on parental and non-parental tumors and have a phospholipid metabolism proton NMR spectroscopy signature
}

\author{
PATRICK MERLE ${ }^{1}$, DANIEL MORVAN ${ }^{2-4}$, JEAN CLAUDE MADELMONT ${ }^{2}$, \\ DENIS CAILLAUD ${ }^{1,4}$ and AICHA DEMIDEM ${ }^{2}$
}

\author{
${ }^{1}$ CHU Clermont-Ferrand, Service de Pneumologie, Hôpital Gabriel Montpied, rue Montalembert, \\ F63001 Clermont-Ferrand; ${ }^{2}$ INSERM 484, rue Montalembert, 63005 Clermont-Ferrand Cedex; \\ ${ }^{3}$ Centre de Lutte Contre le Cancer Jean Perrin, rue Montalembert, 63011 Clermont-Ferrand Cedex; \\ ${ }^{4}$ Univ Clermont 1, 9 Bvd. F. Mitterrand, 63001 Clermont-Ferrand, France
}

Received January 12, 2006; Accepted March 10, 2006

\begin{abstract}
We recently showed, using a parental double B16 melanoma tumor model that, in the presence of CENU-treated primary tumors, untreated secondary tumors exhibited growth inhibition. This response was shown to be related to CENUinduced bystander effects. To see whether CENU-induced bystander effects were still effective on non-parental syngeneic secondary tumors, Lewis lung (3LL) secondary tumors were inoculated in recipients bearing CENU-treated B16 melanoma tumors. Our results show that non-parental secondary $3 \mathrm{LL}$ tumors underwent growth inhibition, differentiation, and phospholipid metabolism alterations, all changes similar to those of parental secondary 3LL tumors. This demonstrates the lack of tumor tissue specificity of chemotherapy-induced bystander effects.
\end{abstract}

\section{Introduction}

The main cytotoxic effect of chloroethylnitrosourea (CENU) is attributed to DNA damage $(1,2)$. However, beside DNA damage, we previously reported that, in the presence of a primary CENU-treated tumor, parental secondary untreated tumors implanted a long time after CENU treatment and at a remote location from treated tumors underwent growth inhibition, differentiation and altered metabolic profile. These phenotypes changes were similar to those of treated tumors (3). These findings allowed us to demonstrate that primary

Correspondence to: Dr Aicha Demidem, INSERM U484, rue Montalembert, 63005 Clermont-Ferrand, France

E-mail: demidem@inserm484.u-clermont1.fr

Key words: chloroethylnitrosoureas, bystander effects, secondary tumors, proton NMR spectroscopy, phospholipid metabolism
CENU-treated tumors released a factor having inhibitory properties on distant secondary untreated tumors (4). We named this phenomenon the chemotherapy-induced bystander effect, in analogy with the similar phenomenon in ionizing radiation studies (4-6). Some proteins were released by CENUtreated B16 melanoma cells, such as phosphatidylethanolaminebinding protein, cardiovascular heat-shock protein, Rhoassociated coiled-coil forming kinase 2 and actin fragments, which accompanied the expression of the bystander factors (4).

To see whether this bystander effect was specific to parental tumors or could also occur on non-parental tumors, we used double tumor models involving secondary untreated 3LL tumors with either parental 3LL carcinoma or non-parental B16 melanoma CENU-treated primary tumors. We show that, in the presence of CENU-treated primary B16 melanoma tumors, non-parental secondary untreated 3LL tumors dramatically reduced their proliferation and underwent histological changes (tumor necrosis, decreased number of mitosis and vascular density), and metabolic alterations. All of these modifications were similar to phenotype changes of parental secondary 3LL tumors, and to CENU-treated 3LL tumors. Metabolite profiling of the response using proton HRMAS NMR spectroscopy demonstrated alterations in the phospholipid (Plp) metabolism, including an increase in phosphoethanolamine (PE) in B16 melanoma (4) and a decrease in glycerophosphocholine (GPC) in 3LL carcinoma.

In conclusion, this article provides evidence of effective bystander effects on tumors expressing different tumor antigens, and of a signature of these effects on the tumor phospholipid metabolism.

\section{Materials and methods}

Chemical. A member of the CENU drug family, cystemustine [N'-(2-chloroethyl)-N-(2-(methylsulphonyl)-ethyl)-N'-nitrosourea] (Orphachem, Clermont-Ferrand, France) (7) was used in experiments, and was prepared as $5 \mathrm{mM}$ solution in $0.9 \%$ $\mathrm{NaCl}$ before being used in vivo by injection into mice. 
Cell culture. The transplantable B16 (F1) melanoma cells and Lewis lung carcinoma cells (3LL) originating from C57BL6/6J Ico mice were obtained from ICIG (Villejuif, France) and adapted to grow in culture. The cells were maintained as monolayers in culture flasks using culture medium consisting of Eagle's MEM-glutaMAX medium (Life Technologies). Cells were grown in a humidified atmosphere at $37^{\circ} \mathrm{C}$ under $5 \% \mathrm{CO}_{2}$.

In vivo models. Six- to eight-week-old C57BL6/6J male mice were purchased from IFFA CREDO, France. All procedures were approved by the Animal Experimental Ethics Committee. Mice were shaved before s.c. injection into their flanks of $5 \times 10^{5}$ tumor cells (B16 melanoma or 3LL cells). B16 melanoma tumors or 3LL tumors became palpable at days 8-10 after cell inoculation. Four groups of mice (20 recipients per groups) were used, untreated groups which received B16 cell or 3LL cell injection, followed by sham injections of saline solution, and CENU-treated groups which received B16 cell or 3LL cell injection followed by CENU administration at a dose of $15 \mu \mathrm{g} / \mathrm{g}$ body weight. CENU was injected intratumorally, at days 11, 14 and 18 from cell inoculation. Tumors were removed, dissected, weighed and prepared for histology and ex vivo NMR spectroscopy examinations.

Double tumor models: challenge with $3 L L$ cells. Mice bearing CENU-treated tumors were used to assess the effect of prime CENU treatment on the fate of secondary untreated parental and non-parental tumors. Two groups of animals were conducted (24 recipients per groups), one bearing B16 melanoma CENUtreated tumors, the other bearing 3LL CENU-treated tumors. On day 30 , each group of mice was challenged on the opposite flank with $5 \times 10^{5}$ 3LL untreated cells. Secondary tumor development and growth were evaluated twice a week.

Primary and secondary tumors were followed until day 56. Naive groups of mice were injected with B16 melanoma or $3 \mathrm{LL}$ cells at the same time. Tumor surface areas were calculated by measuring the length and width, with a caliper square (8). At different times of evolution, three mice of each group were sacrificed. We defined a growth inhibition ratio as the mean over time of the ratio of secondary untreated tumor mass to control tumor mass.

Histological analyses. Tumor pieces were fixed in a formol solution. Paraffin-embedded sections were cut into $4-\mu \mathrm{m}$ sections, and tissue sections were prepared for hematoxylin and eosin staining and routine pathological analysis. The count of mitoses was performed on 10 microscopic fields (HPFs). Other histological evaluation included necrosis area and vasculature. The vascular density index was quantified on 5 microscopic fields with a scale from 0 to $3(0$, nonsignificant; 1, small; 2, moderate; 3 , large).

Proton high resolution magic angle spinning (HRMAS) NMR spectroscopy. Proton HRMAS NMR spectroscopy was performed at $500 \mathrm{MHz}$ (Bruker DRX 500). Samples, consisting in pieces of intact tumor tissue below $50 \mathrm{mg}$, were inserted into 4-mm diameter zirconia rotors and rotated at $4 \mathrm{kHz}$.

Metabolite profiling was performed based on a conventional one-dimensional proton saturation recovery sequence (repetition time, $10 \mathrm{sec}$; spectral width, $10 \mathrm{ppm}$; complex data points, $16 \mathrm{~K}$; number of samples, 64; water signal presaturation at low power). Quantification was performed on an $\mathrm{O} 2$ workstation (Silicongraphics) using the built-in deconvolution software (XWIN-NMR v2.6, Bruker), and normalization was performed relative to the water signal. Measured derivatives from one-dimensional spectra included phosphatidylcholine (PtdCho) $\left(\left[\mathrm{N}\left(\mathrm{CH}_{3}\right)_{3}\right]\right.$ broad signal at $\left.3.26 \mathrm{ppm}\right)$, taurine (Tau) $\left(\mathrm{CH}_{2}\right.$ signal at $\left.3.43 \mathrm{ppm}\right)$, glycine (Gly) $\left(\mathrm{CH}_{2}\right.$ signal at $3.56 \mathrm{ppm})$, lysine (Lys) $\left(\varepsilon-\mathrm{CH}_{2}\right.$ signal at $\left.3.00 \mathrm{ppm}\right)$, total creatine $(\mathrm{tCr}=$ creatine + phosphocreatine $)\left(\mathrm{CH}_{3}\right.$ signal at $3.03 \mathrm{ppm})$, total gluthatione $(\mathrm{GSx}=$ reduced + oxidized glutathione) $\left(\mathrm{CH}_{2}\right.$ signal at $\left.2.55 \mathrm{ppm}\right)$, lactate $(\mathrm{Lac})\left(\mathrm{CH}_{3}\right.$ signal at $1.33 \mathrm{ppm})$, and alanine (Ala) $\left(\mathrm{CH}_{3}\right.$ signal at $\left.1.47 \mathrm{ppm}\right)$. Glycerophosphocholine (GPC) was identified on onedimensional proton spectra by the chemical shifts of its choline moiety at $3.23 \mathrm{ppm}\left[\mathrm{N}\left(\mathrm{CH}_{3}\right)_{3}\right], 3.68 \mathrm{ppm}(\mathrm{CHB} 2-\mathrm{N})$, and $4.34 \mathrm{ppm}\left(\mathrm{O}-\mathrm{CH}_{2}\right)$. One-dimensional profiling was completed by two-dimensional profiling using proton total correlation spectroscopy (TOCSY) sequence (repetition time, $1.5 \mathrm{sec}$; spectral width, 6x6 ppm; complex data points, $2 \mathrm{~K}$ x 256; number of samples, 16; spin mixing time, $75 \mathrm{~ms}$; water signal presaturation at low power). TOCSY spectra were processed using XWIN-NMR software. Measured derivatives included GPC (signal at $3.68 \times 4.34 \mathrm{ppm}$ ), glycerophosphoethanolamine (GPE) (signal at 3.30x4.12 ppm), ethanolamine (E) (signal at $3.15 \times 3.80 \mathrm{ppm}$ ), phosphoethanolamine (PE) (signal at $3.22 \times 3.99 \mathrm{ppm}$ ), choline (Cho) (signal at 3.55x4.07 ppm), and phosphocholine (PC) (signal at $3.62 \times 4.18 \mathrm{ppm}$ ). Quantification was performed using quantitative TOSCY coupled with onedimensional saturation recovery as previously described (9).

Statistical analysis. Data are presented as mean \pm standard deviation. Comparisons between groups were performed using the Mann-Whitney test. Time series were analysed using ANOVA, followed by a post hoc test (SEM software) (10) with paired comparisons between groups.

\section{Results}

Tumor mass measurements of untreated and CENU-treated tumors

Untreated tumors. Both untreated B16 melanoma or 3LL tumors showed exponential growth at day 25, the untreated tumors reached a weight of $4.6 \pm 0.91 \mathrm{~g}$ and $7.3 \pm 1.5 \mathrm{~g}$ for B16 melanoma and $3 \mathrm{LL}$ tumors respectively $\left(\mathrm{p}<10^{-6}\right.$ ) (data not shown). In these untreated groups, tumor size measurements were only available until day 28 since the animals died shortly after.

CENU-treated tumors. CENU-treated B16 tumor proliferation exhibited two phases, a growth-inhibition phase between days 0 and 28 followed by a growth recovery and a plateau phase characterized by tumor weights much lower than those of untreated tumors with a day 25 tumor mass of $0.29 \pm 0.08 \mathrm{~g}$ vs $4.6 \pm 2.7 \mathrm{~g}, \mathrm{p}<10^{-7}$ (treated vs untreated tumors) (data not shown).

CENU-treated 3LL tumors exhibited an initial proliferation from days 11 to 14 , followed by a growth inhibition phase for $17 \pm 3$ days (from days 18 to 35 ) and then by proliferation recovery with a plateau phase. Tumor masses of CENU-treated 


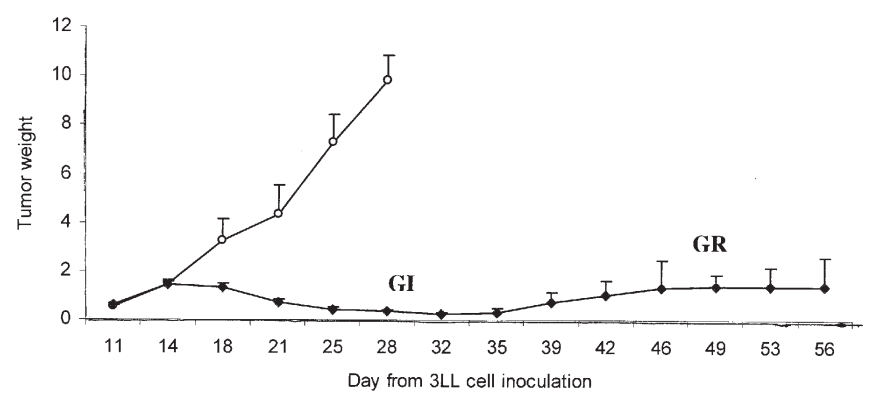

Figure 1. Growth curve of untreated 3LL tumors versus CENU-treated tumors. Tumor weight is given in grams. Untreated 3LL tumors exhibit a roughly exponential growth $(0)$. 3LL cells $\left(5 \times 10^{5}\right)$ were inoculated s.c. at day 0 . In the treated 3LL group $(\bullet), 15 \mu \mathrm{g} / \mathrm{g}$ CENU was injected directly (i.t.) into the tumor at days 11,14 and 18 after cell inoculation. CENUtreated 3LL tumors exhibited an initial proliferation from days 11 to 14 followed by a growth inhibition phase (GI) for days 18-35 and then a growth recovery phase $(\mathrm{GR})$ with a plateau phase.

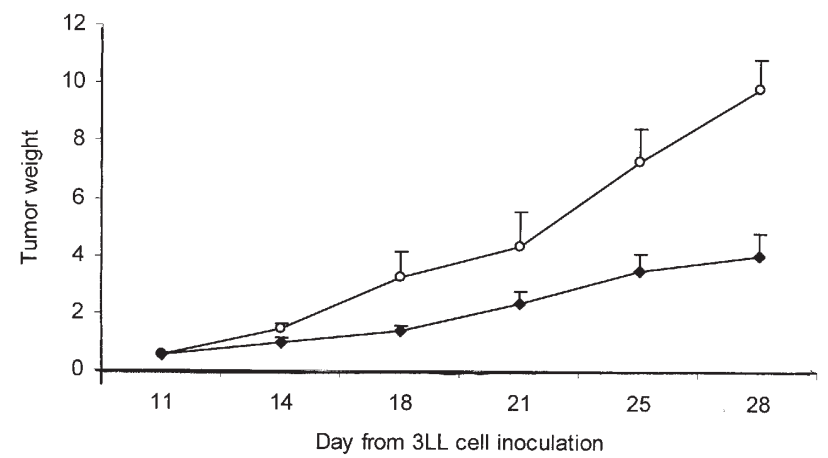

Figure 2. Growth curve of secondary 3LL tumors in recipients bearing primary CENU-treated 3LL tumors. Tumor weight is given in grams. 3LL cells $\left(5 \times 10^{5}\right)$ were inoculated s.c. at day 0 . Primary 3LL tumors were treated by $15 \mu \mathrm{g} / \mathrm{g}$ CENU at days 11,14 and 18 intratumorally. At day 30, mice were challenged on the opposite flank with $5 \times 10^{5} 3 \mathrm{LL}$ cells. Tumor weights were followed until day 28 for control $3 \mathrm{LL}$ tumors (o) and day 58 for primary CENU-treated 3LL tumors, corresponding to day 28 for secondary untreated 3LL tumors $(\bullet)$. The evolution of the tumor mass of secondary $3 \mathrm{LL}$ tumors $(\bullet)$ in these recipients was strongly reduced in comparison with untreated 3LL tumors. ( $\mathrm{p}<10^{-7}$; MANOVA test).

3LL tumors at day 25 were of $0.44 \pm 0.2 \mathrm{~g}$ vs $7.3 \pm 1.5 \mathrm{~g}, \mathrm{p}<10^{-7}$ (treated vs untreated tumors), as shown in Fig. 1. In all experiments, no important toxicity was observed in any of the animals as assessed by weight loss.

Fate of secondary untreated $3 L L$ tumors in primed CENUtreated tumor recipients

Parental secondary tumors. First, we studied the fate of secondary untreated 3LL tumors implanted in primed 3LL CENU-treated recipients. Untreated secondary tumors implanted 30 days after the primary tumor exhibited a strong decrease in tumor mass. Their mass levelled at day 25 at $3.4 \pm 0.5 \mathrm{~g}$ vs $7.3 \pm 1.5 \mathrm{~g}, \mathrm{p}<10^{-7}$ (untreated secondary vs control tumors) (Fig. 2). The growth inhibition ratio of secondary untreated 3LL tumors vs 3LL control tumors was $2.3\left(\mathrm{p}=10^{-7}\right)$.

Non-parental secondary tumors. Second, we investigated the fate of secondary non-parental tumors. Recipients bearing primary CENU-treated B16 melanoma tumors were challenged

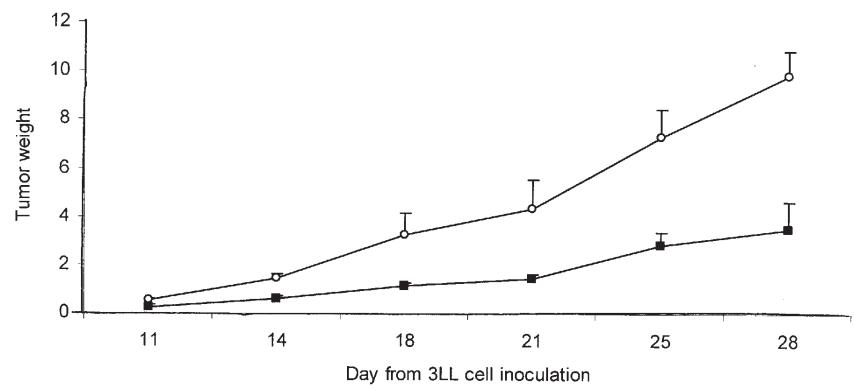

Figure 3. Growth curve of secondary 3LL tumors in recipients bearing primary CENU-treated B16 melanoma tumors. Tumor weight is given in grams. B16 cells $\left(5 \times 10^{5}\right)$ were inoculated s.c. at day 0. Primary B16 tumors were treated by $15 \mu \mathrm{g} / \mathrm{g}$ CENU at days 11,14 and 18 intratumorally. At day 30 , mice were challenged on the opposite flank with $5 \times 10^{5} 3 \mathrm{LL}$ cells. Tumor mass of secondary untreated 3LL tumors ( $\mathbf{a})$ in recipients bearing primary CENU-treated B16 tumors was strongly reduced in comparison with untreated 3LL tumors $(0)\left(\mathrm{p}<10^{-7}\right.$; MANOVA test).

with 3LL carcinoma cells. The growth curve of secondary 3LL tumors is presented in Fig. 3. The proliferation was inhibited in the presence of CENU-treated B16 tumors. At day 25, tumor masses were evaluated at $2.85 \pm 0.8 \mathrm{~g}$ vs $7.3 \pm 1.5 \mathrm{~g}, \mathrm{p}<10^{-7}$, secondary untreated tumors vs control tumors. The overall growth inhibition ratio of secondary untreated 3LL tumors vs 3LL control tumors was $3.07\left(\mathrm{p}=10^{-7}\right)$.

Histological analyses. In 3LL CENU-treated tumors, the inhibition of growth was accompanied by several histological modifications. We examined the following features: number of mitoses, necrosis area and vascular density index.

In 3LL control tumors, the count of mitoses per $10 \mathrm{HPFs}$ between days $17-24$ was $74 \pm 12$. The vascular density index was high (index $=3 \pm 0.6$ ) (Fig. 4A).

CENU-treated 3LL tumors showed alteration in morphology, large cells with polyploidy, vascular thrombosis and fibrosis. The mitosis count between days 18-28 dropped to $24 \pm 21$ mitoses per 10 HPFs. The vascular density index was strongly reduced (index $=1 \pm 0.6$ ), and necrosis areas were more pronounced (Fig. 4B).

The histology of secondary untreated 3LL tumors in recipients bearing primary CENU-treated 3LL tumors or B16 tumors revealed similar modifications than those found in CENU-treated tumors, including low vascular density index (index $=1 \pm 0.3$ ), vascular thrombosis and fibrosis (Fig. 4C and D).

Tumor metabolite profiling using proton HRMAS NMR spectroscopy

Primary treated 3LL tumors. The metabolite profiles of 3LL untreated tumors were compared to those of 3LL CENU-treated tumors. Data show that, during the growth inhibition phase (between days 18-28), a strong decrease in glycerophosphocholine (GPC) $(\mathrm{p}<0.02$, primary tumors vs control tumors), glycerophosphoethanolamine $(\mathrm{GPE})(\mathrm{p}<0.05)$ and glycine (Gly) $(\mathrm{p}<0.03)$ levels, and ethanolamine $(\mathrm{E})$ content $(\mathrm{p}<0.03)$ occurred. Other metabolites, such as alanine (Ala) and total creatine (tCr), increased while lysine (Lys) and taurine (Tau) levels were not modified. During the recovery phase, there was a return to the baseline of most of these variations but not GPC and GPE (p<0.05) (Fig. 5). 


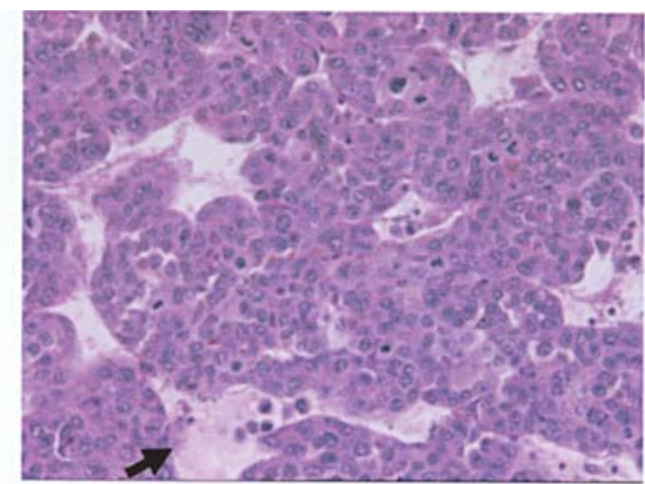

A

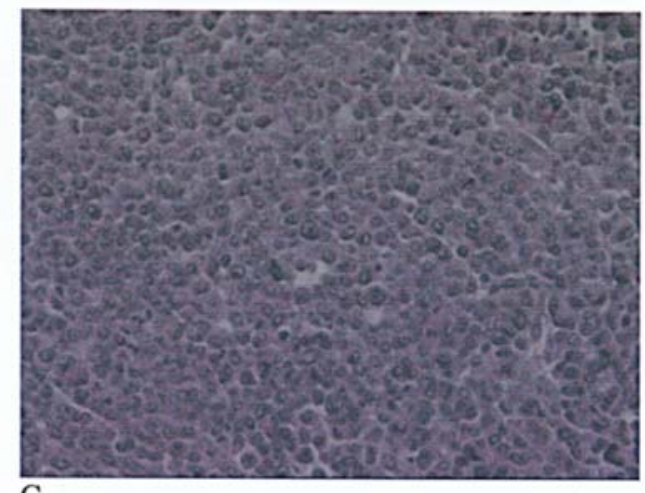

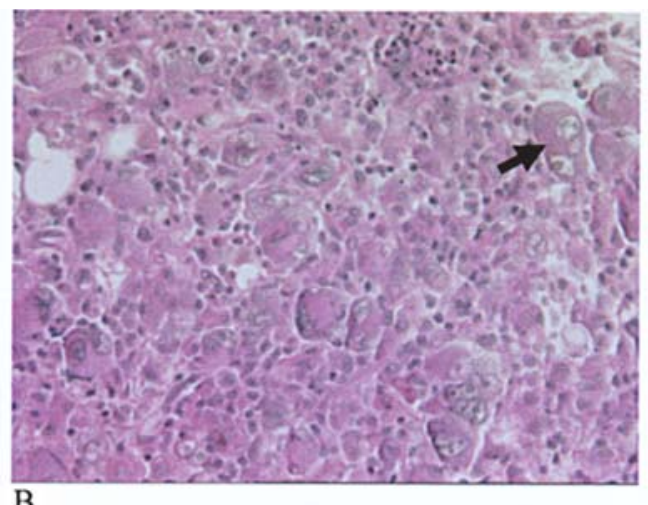

B

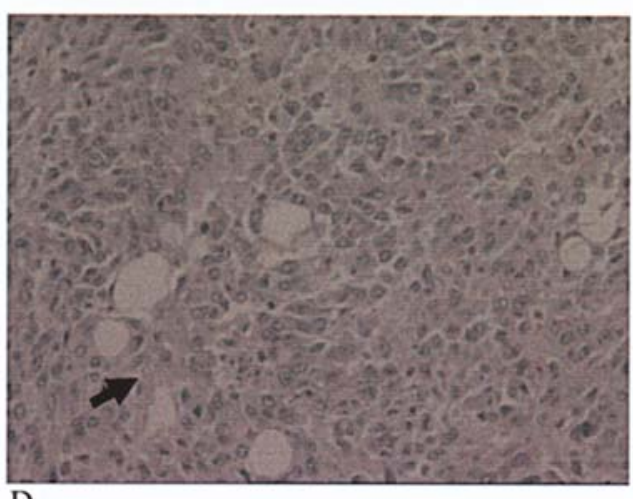

$\mathrm{D}$

Figure 4. Histological features of 3LL tumors. A, untreated 3LL tumor, 17 days after cell inoculation; the count of mitosis per 10 HPFs was $74 \pm 12$, histology

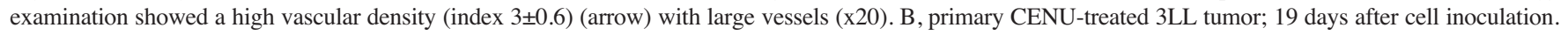
The mitosis count dropped to $24 \pm 21$ mitoses per $10 \mathrm{HPFs}$. The morphology of cells was altered with a decrease in aggressiveness, large cells with polyploidy (arrow), reduction of the vascular density index at $1 \pm 0.6$, necrosis areas and fibrosis $(\mathrm{x} 20)$. C and D, secondary untreated $3 \mathrm{LL}$ tumor in a recipient bearing a primary CENU-treated 3LL tumor (C) or a B16 melanoma tumor (D). Examination revealed similar histopathological modifications to those found in primary CENU-treated 3LL tumors. Prominent features in both parental and non-parental secondary tumors concerned low vascular density index (index $=1 \pm 0.3$ ), thrombosis and fibrosis (arrow) (x20).

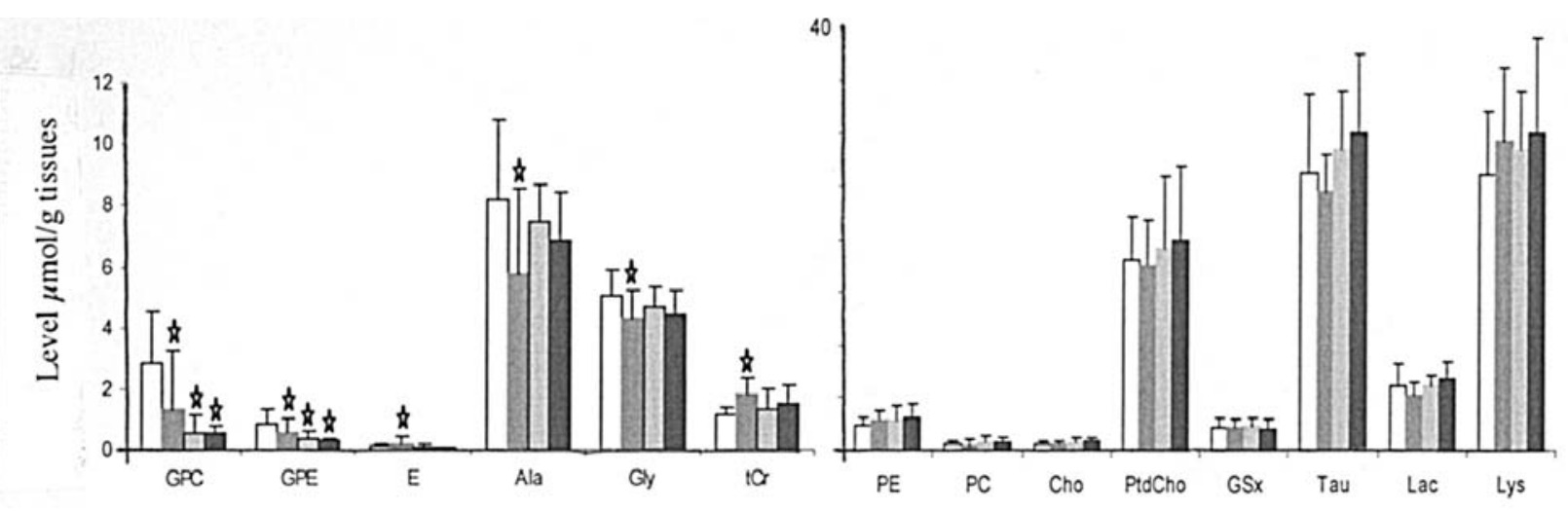

Figure 5. Proton HRMAS NMR spectroscopy metabolite profiling of 3LL untreated tumors (white bars), 3LL CENU-treated tumors during the growth inhibition phase (dark grey bars), 3LL CENU-treated tumors during the recovery phase (light grey bars), and contemporary 3LL secondary tumor (black bars). Measured derivatives included phosphatidylcholine (PtdCho), taurine (Tau), glycine (Gly), lysine (Lys), total creatine (tCr), total gluthatione (GSx), lactate (Lac), alanine (Ala), glycerophosphocholine (GPC), glycerophosphoethanolamine (GPE), ethanolamine (E), phosphoethanolamine (PE), choline (Cho), phosphocholine (PC). ${ }^{*} \mathrm{P}<0.05$.

Secondary $3 L L$ tumor. Secondary untreated 3 LL tumors implanted in recipients bearing either parental or non-parental tumors exhibited similar metabolic changes encompassing significant GPC and GPE decrease $(\mathrm{p}<0.05)$. This metabolite profile was similar to that of primary CENU-treated 3LL tumors (Fig. 6).

\section{Discussion}

CENU treatment was able to induce modifications other than DNA damage, such as the acquisition of new phenotype (Plp metabolism, differentiation, loss of aggressiveness) (11). Also CENU treatment induced growth inhibition of parental 
A
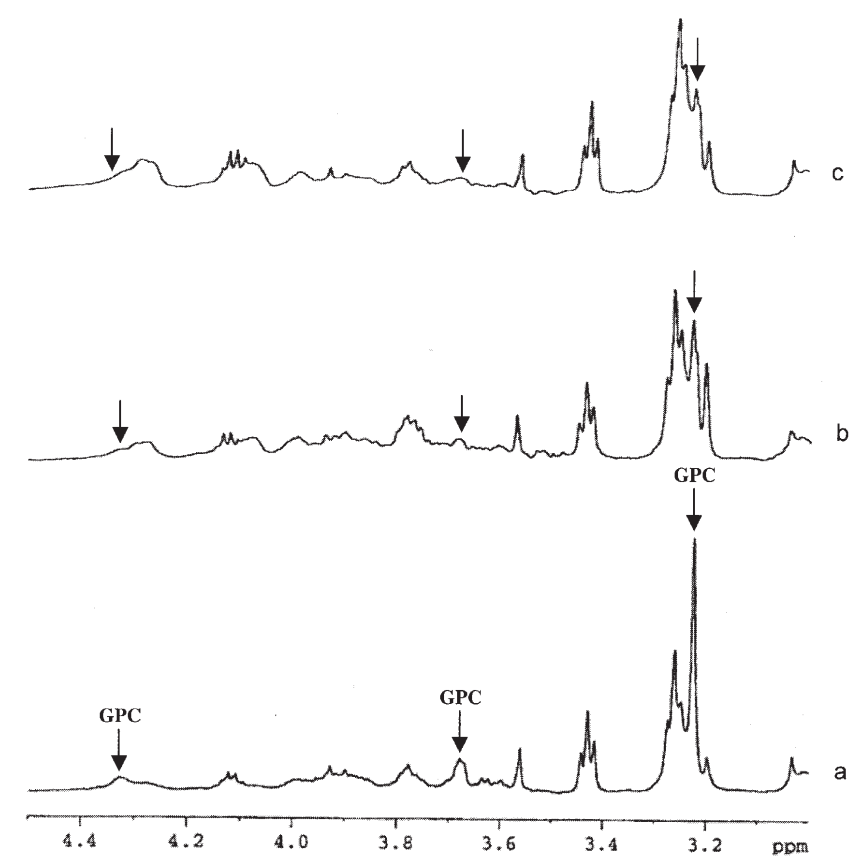

B
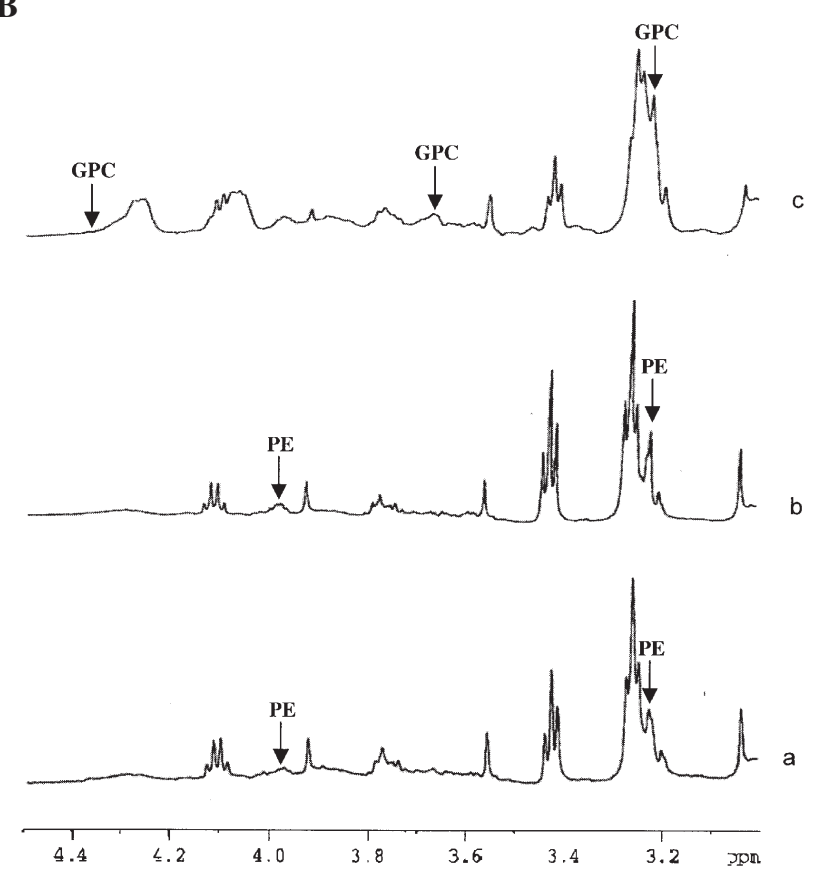

Figure 6. A, proton HRMAS NMR spectra in a parental secondary 3LL tumor. Typical NMR spectra of (a) untreated 3LL tumor at day 18; (b) primary CENU-treated 3LL tumor at day 39; and (c) contemporary secondary untreated 3LL tumor. CENU-treated 3LL tumor and secondary untreated tumor show a strong decrease in GPC, in comparison with the 3LL control tumor. B, proton HRMAS NMR spectra in a non-parental secondary 3LL tumor. Typical NMR spectra of (a) untreated B16 tumor at day 18; (b) primary CENU-treated B16 tumor at day 39; and (c) contemporary secondary untreated 3LL tumor. Comparison between untreated B16 tumor and CENU-treated B16 tumors shows an increase in PE in primary CENU-treated B16 tumor and a decrease in GPC in secondary untreated 3LL tumor vs 3LL control tumor (A).

secondary tumors in a melanoma murine model (3). This latter effect was mediated by bystander factors (4). Thus, we asked whether this bystander effect was specific to parental tumors or may occur also on non-parental tumors. In the present study, we used double tumor models of parental and non-parental secondary untreated tumors, including syngenic 3LL carcinoma. We found that secondary 3LL tumors underwent the same phenotype changes whatever the tumor type of the primary treated tumor. A bystander effect inducted by interleukin 12 gene transduction was reported by Adris et al (12), and was mediated by an immune response against parental and non-parental tumors in a syngenic model. Mice vaccinated with IL-12 gene-transduced CT26 colon cancer cells developed a long-lasting antitumor immune memory able to reject not only parental cells but also syngeneic LM3 mammary and MCE fibrosarcoma cells. After tumor cell inoculation, mice developed local nodules for a few days, which then ceased growing and regressed. These nodules contained a necrotic central area and a peripheral area containing blood vessels, active fibroblasts, and neutrophils. The high density of blood vessels suggested that an angiogenic process was taking place.

Histological analyses revealed an impact of CENU treatment on B16 or 3LL tumor micro-vessel content, a decreased vascularisation density, together with a reduction of mitoses and a loss of aggressiveness. Parental secondary untreated B16 melanoma or non-parental secondary 3LL tumors showed similar histopathological alteration with a dramatic impact on tumor vascularisation, suggesting an anti-angiogenic effect of bystander factors. The differentiation pattern, involving mitosis reduction and loss of aggressiveness, was found both in secondary and primary tumors.
During the growth inhibition period, treated 3LL tumors exhibited alterations in Ala, Gly, and $\mathrm{tCr}$ that may be related to the energy metabolism. A decrease in Gly together with an increase in $\mathrm{tCr}$ suggests an increased $\mathrm{tCr}$ biosynthesis possibly to increase the storage of high energy bonds of ATP. The decrease in Gly and Ala, together with the maintenance of Lac, may suggest an alteration in pyruvate biosynthesis or consumption.

Another group of metabolite changes related to the phospholipid metabolism. In control 3LL tumors, there was an accumulation of GPC. Previous reports showed that tumor growth regulation and cell survival tightly involves Plp metabolism. Apoptosis has been shown to be mediated by a drop in PtdCho level or to a mutation in choline cytidylyl transferase (CCT), the key enzyme of the cytidine diphosphate (CDP)-pathway $(13,14)$. In the 3LL model, there is an excess of phosphatidylcholine in lung carcinoma compared to adenocarcinoma in other organs (15) that could explain the high GPC level in 3LL controls. Phospholipid accumulation was also associated with the inhibition of cell proliferation and/or the arrest of the cell cycle. To maintain membrane phospholipid homeostasis, in response to an excess of phospholipid, phospholipase A2 was shown to be activated, yielding to a GPC accumulation (16).

During the growth inhibition phase, CENU treatment of 3LL tumors induced a decrease of GPC and GPE concentration. During the recovery phase, most metabolic alteration returned to control values, except GPC and GPE. This was consistent with alterations in membrane metabolism with a decrease of membrane degradation products (17-19), a decrease of cytosolic phospholipase $A_{2}$ expression (20), the inhibition of 
excessive Plp biosynthesis or increased Plp consumption (16). Such persistent phospholipid metabolism alteration was also observed in CENU-treated primary B16 and untreated secondary B16 melanoma tumors, mainly consisting of an increase in PE $(4,11)$. PE was a metabolic marker found in other types of tumor cells when they escaped therapy-induced cell death, and could be a mediator of cell survival in tumors $(11,21)$.

In conclusion, CENU treatment of a primary tumor was able to induce growth inhibition, tumor cell differentiation and Plp metabolism alterations of a non-parental secondary untreated tumor. This demonstrates the lack of tumor cell type specificity (antigens) of chemotherapy-induced bystander effects, and of the signature of these effects on phospholipid metabolism.

\section{Acknowledgments}

The authors thank Monique De Latour for histopathology analyses, and Janine Papon for her technical assistance. This study was supported by the Institut National de la Santé et de la Recherche Médicale (INSERM). The authors declare that they have no competing financial interests.

\section{References}

1. Ewig RA and Kohn KW: DNA damage and repair in mouse leukemia L1210 cells treated with nitrogen mustard, 1,3-bis(2chloroethyl)-1-nitrosourea, and other nitrosoureas. Cancer Res 37: 2114-2122, 1977.

2. Brent TP, Lestrud SO, Smith DG and Remack JS: Formation of DNA interstrand cross-links by the novel chloroethylating agent 2-chloroethyl(methylsulfonyl)methanesulfonate: suppression by O6-alkylguanine-DNA alkyltransferase purified from human leukemic lymphoblasts. Cancer Res 47: 3384-3387, 1987.

3. Demidem A, Morvan D, Papon J, De Latour M and Madelmont JC: Cystemustine induces redifferentiation of primary tumors and confers protection against secondary tumor growth in a melanoma murine model. Cancer Res 61: 2294-2300, 2001.

4. Demidem A, Morvan D and Madelmont JC: Bystander effects are induced by CENU treatment and associated with altered protein secretory activity of treated tumor cells: A relay for chemotherapy? Int J Cancer (In press).

5. Lyng FM, Seymour CB and Mothersill C: Initiation of apoptosis in cells exposed to medium from the progeny of irradiated cells: a possible mechanism for bystander-induced genomic instability? Radiat Res 157: 365-370, 2002.
6. Ohba K, Omagari K, Nakamura T, et al: Abscopal regression of hepatocellular carcinoma after radiotherapy for bone metastasis, Gut 43: 575-577, 1998.

7. Madelmont JC: Cystemustine. Drugs Future 19: 27-30, 1994.

8. Dolan ME, Moschel RC and Pegg AE: Depletion of mammalian O6-alkylguanine-DNA alkyltransferase activity by O6-benzylguanine provides a means to evaluate the role of this protein in protection against carcinogenic and therapeutic alkylating agents. Proc Natl Acad Sci USA 87: 5368-5372, 1990.

9. Morvan D, Demidem A, Papon J and Madelmont JC: Quantitative HRMAS proton correlation spectroscopy applied to cultured melanoma cells treated by chloroethyl-nitrosourea: demonstration of phospholipid metabolism alterations. Magn Reson Med 49: 241-248, 2003.

10. Kwiatkowski F, Girard M, Hacene K and Berlie J: Sem: a suitable statistical software adaptated for research in oncology. Bull Cancer 87: 715-721, 2000.

11. Morvan D, Demidem A, Papon J, De Latour M and Madelmont JC: Melanoma tumors acquire a new phospholipid metabolism phenotype under cystemustine as revealed by high-resolution magic angle spinning proton nuclear magnetic resonance spectroscopy of intact tumor samples. Cancer Res 62: 1890-1897, 2002.

12. Adris S, Chuluyan E, Bravo A, et al: Mice vaccination with interleukin 12-transduced colon cancer cells potentiates rejection of syngeneic non-organ-related tumor cells. Cancer Res 60: 6696-6703, 2000.

13. Cui Z, Houweling M, Chen MH, Record M, Chap H, Vance DE and Tercé FA: Genetic defect in phosphatidylcholine biosynthesis triggers apoptosis in Chinese Hamster Ovary cells. J Biol Chem 271: 14668-14671, 1996.

14. Mallampalli RK, Ryan AJ, Salom RG and Jackowski S: Tumor necrosis factor-alpha inhibits expression of CTP:phosphocholine cytidylyltransférase. J Biol Chem 275: 9699-9708, 2000.

15. Itoh K, Nakamura M and Akino T: Comparison of phospholipid profiles of primary adenocarcinoma in the lung and other organs. Lipids 12: 876-880, 1981

16. Baburina I and Jackowski S: Cellular response to excess phospholipid. J Biol Chem 274: 9400-9408, 1999.

17. McPhail LD, Chung YL, Madhu B, Clark S, Griffiths JR, Kelland LR and Robinson S: Tumor dose response to the vascular disrupting agent 5,6-dimethylxanthenome-4-acetic acid, using in vivo magnetic resonance spectroscopy. Clin Cancer Res 11: 3705-3713, 2005.

18. De Jong S, Mulder NH, De Vries EGC and Robillard GT: NMR spectroscopy analysis of phosphorus metabolites and the effect of adriamycin on these metabolite levels in an adriamycinsensitive and resistant human small cell lung carcinoma cell line. Br J Cancer 63: 205-212, 1991.

19. Griffin JL and Shockcor JP: Metabolic profiles of cancer cells. Nat Rev 4: 551-561, 2004.

20. Glunde K, Chunfa J and Bhujwalla ZM: Molecular causes of the aberrant choline phospholipid metabolism in breast cancer. Cancer Res 64: 4270-4276, 2004.

21. Kiss Z: Regulation of mitogenesis by water-soluble phospholipid intermediates. Cell Signal 11: 509-514, 1999. 